\title{
Comparison of Hernia Recurrence and Seroma Formation in Cancer Patients Undergoing Incisional Hernia Repair with Transfascial Sutures vs. Fibrin Glue for Mesh Fixation
}

\author{
Celia Ledet ${ }^{1, \text {, }}$, David Santos ${ }^{1}$, Xuemei Wang ${ }^{2}$, Heather Gibson $^{1}$, Angela Limmer ${ }^{1}$, Brian Badgwell ${ }^{1}$ \\ ${ }^{1}$ Departments of Surgical Oncology, The University of Texas MD Anderson Cancer Center, Houston, Texas, USA \\ ${ }^{2}$ Departments of Biostatistics, The University of Texas MD Anderson Cancer Center, Houston, Texas, USA
}

Email address:

CRobinson3@mdanderson.org (C. Ledet)

${ }^{*}$ Corresponding author

To cite this article:

Celia Ledet, David Santos, Xuemei Wang, Heather Gibson, Angela Limmer, Brian Badgwell. Comparison of Hernia Recurrence and Seroma Formation in Cancer Patients Undergoing Incisional Hernia Repair with Transfascial Sutures vs. Fibrin Glue for Mesh Fixation. International Journal of Clinical Oncology and Cancer Research. Vol. 6, No. 1, 2021, pp. 42-48. doi: 10.11648/j.ijcocr.20210601.17

Received: February 15, 2021; Accepted: February 25, 2021; Published: March 4, 2021

\begin{abstract}
Background: Open ventral incisional hernia repairs with mesh reduce hernia recurrence rates to less than 10\%. The Rives-Stoppa retrorectus hernia repair technique is becoming the standard of care for mesh repair of complex incisional hernias. In these hernia repairs, mesh has traditionally been fixated with transfascial sutures, but fibrin glue has been used as an alternative to reduce pain and may also reduce operative time and the risk of seroma formation. The primary objective of this study was to compare the rates of hernia recurrence and seroma formation in cancer patients who underwent Rives-Stoppa incisional hernia repair using transfascial sutures vs. fibrin glue for mesh fixation. Methods: We conducted a retrospective review of a prospectively maintained database of cancer patients at our free-standing cancer institution who underwent Rives-Stoppa ventral incisional hernia repair performed at our institution. Fisher's exact test was used to assess differences in hernia recurrence and seroma formation rates between patients who underwent mesh fixation with transfascial sutures vs. fibrin glue. Using a neutral prior probability distribution, a Bayesian analysis was performed to evaluate the posterior probability of seroma formation requiring intervention and hernia recurrence after retrorectus repair with fibrin glue fixation. Log-rank test was used to assess the difference in overall survival between the two groups. Results: Forty-one patients ( 22 in the transfascial suture group and 19 in the fibrin glue group) were included in the analysis. The patient demographics were similar in both groups. There was no statistically significant difference in seroma formation requiring drainage by interventional radiology (IR) between the fibrin glue and the transfascial suture groups $(5.3 \%$ vs. $9.1 \% ; P=1.00)$. The odds of having a hernia recurrence was 4 times higher in the transfascial suture group than in the fibrin glue fixation group, but this was not statistically significant $(18.2 \% \mathrm{vs} 5.3 \%$, $\mathrm{p}=0.35)$. The Bayesian analysis showed a $90 \%$ probability that the hernia recurrence rate would be higher in the transfascial suture than in the fibrin glue fixation group. Conclusion: Using fibrin glue instead of transfascial sutures may reduce hernia recurrences and seroma formation in cancer patients undergoing ventral incisional hernia repairs with retro rectus mesh. The high probability of reducing the odds of hernia recurrence with fibrin glue fixation warrants a prospective randomized, multi-institutional clinical trial.
\end{abstract}

Keywords: Ventral Incisional Hernia, Rives-Stoppa Technique, Fibrin Glue, Seroma, Hernia Recurrence

\section{Introduction}

Incisional ventral hernias are a common complication of the approximately 2 million laparotomies performed in the United States each year, occurring in $2 \%$ to $10 \%$ of patients [1]. As a result, approximately 200,000 incisional ventral hernia repairs are performed annually in the US. [2] Recurrence rates of up to $50 \%$ have been reported for suture-only repairs, but tension-free incisional hernia repairs with the use of prosthetic or biologic mesh have reduced recurrence rates to less than $10 \%$ [3]. Although the use of mesh has helped reduce hernia recurrence rates, it is not without complications. Mesh use has been 
associated with seroma and intestinal fistula formation, chronic pain, infection, and intestinal obstruction. [4, 5] However, incisional hernia repair using the Rives-Stoppa technique, in which mesh is placed in the retro rectus space, has been shown to reduce the incidence of mesh-related complications [6].

Transfascial sutures have traditionally been used to stabilize mesh during tissue incorporation; however, they have been associated with chronic pain. [7] In the 1970s, Chevrel et al. used fibrin glue fixation for fascial closure reinforcement during onlay ventral hernia repairs. [8] Since then, fibrin glue has also been used to fix a mesh to the parietal wall to help eliminate postoperative pain from peritoneal stretching and nerve compression. [9] Studies suggest that fibrin glue fixation may reduce not only pain but also operative time and the risk of seroma formation. [10, 11] Fibrin glues are made from fibrinogen and thrombin, a combination that supports hemostasis and fibroblast proliferation and, eventually, fibrinolysis and incorporation of the mesh into surrounding tissues. [12]

Although there have been additional studies of fibrin glue since the publication of Chevrel's landmark article, it remains unclear whether fibrin glue has similar fixation properties and a similar complication profile to that of transfascial suture fixation. Thus, the aim of our study was to compare the rates of hernia recurrence and seroma formation requiring intervention in cancer patients who underwent open Rives-Stoppa incisional hernia repair using transfascial sutures vs. fibrin glue for mesh fixation.

\section{Methods}

\subsection{Patient Selection}

After receiving approval from The University of Texas MD Anderson Cancer Center's Institutional Review Board, we retrospectively identified all patients who had undergone ventral incisional hernia repairs using the Rives-Stoppa technique, in which polypropylene mesh was placed in a retromuscular position, between August 2016 and August 2018. Patient were excluded if they underwent incisional hernia repair in which mesh was placed in another plane of dissection (i.e. onlay, inlay or intraperitoneal). All procedures were performed by our institution's acute-care general surgery service. Informed consent was waived due to the retrospective nature of this study. The fibrin glue group consisted of patients in whom fibrin glue was used for mesh fixation, and the transfascial suture group consisted of patients in whom traditional transfascial suture fixation was used. Glue versus transfascial fixation was determined based on secular trends and practiced based learning. Data on patient demographics (age, sex, race, body mass index), comorbidities (Type II diabetes mellitus, tobacco use, chronic obstructive pulmonary disease, steroid use), cancer characteristics (cancer type, stage, and use of chemotherapy and/or radiation), perioperative factors (incision location, intraoperative drain placement, surgical approach (open, robotic, or laparoscopic), use of advancement flaps, emergent vs. elective procedure, and combined surgical cases) and postoperative complications (seroma formation requiring drainage by interventional radiology, surgical site infections, hernia recurrences) were collected for all patients.

\subsection{Operative Technique}

Retro muscular hernia repairs were performed in all patients. An additional anterior (anterior component separation) or posterior (transversus abdominus release) advancement flap was needed in some patients to facilitate fascial closure. The retrorectus space was dissected laterally to the semilunar lines, superiorly to the xiphoid, and inferiorly to the pubis. The posterior rectus sheath was closed tension-free with a running polyadiaxonone monofilament absorbable suture. The entire hernia defect was measured in two dimensions and the mesh was then sized to fit into the retromuscular space. Midweight polypropylene, heavy weight polypropylene, acellular porcine dermis mesh types were used. Approximately 10 to $20 \mathrm{~mL}$ of fibrin sealant was used to affix the mesh to the posterior sheath A 15 or 19 French drain was placed above the mesh in the retromuscular position. Then, two continuous, polyadiaxonone sutures were used to close the anterior rectus fascia. Drain output was recorded daily and once output was less than $30 \mathrm{~mL}$ for 72 hours, patients returned to clinic to have the drain removed.

\subsection{Statistical Analysis}

Patient and clinical characteristics were summarized using descriptive statistics. Wilcoxon rank-sum tests and Fisher's exact tests were used to assess the difference in hernia recurrence and seroma formation rates between the fibrin glue and transfascial suture groups. Odds ratios and 95\% confidence intervals were calculated for hernia recurrence and seroma development. Using a neutral non-informative prior distribution, the Bayesian posterior prior probabilities of hernia recurrence and seroma development were individually calculated, along with the $95 \%$ credible intervals. We also computed the posterior probability of one group being higher than the other in terms of the probability of hernia recurrence or seroma development. Statistical significance was defined by a $p$ value of 0.5 .

Overall survival is defined as the time interval between the date of surgery and the date of death due to any cause, or the date of last follow-up whichever occurred first. The probability of overall survival is estimated using the method of Kaplan and Meier. Log-rank test was used to assess the difference in overall survival between the two groups. Statistical analyses were performed using SAS 9.4 [The SAS Institute, Cary, NC].

\section{Results}

Forty-one patients were included in the analysis. There were $22(54 \%)$ patients who underwent incisional hernia repair with transfascial suture fixation (control group) and 19 (46\%) who underwent fibrin glue fixation (experimental 
group). Table 1 shows patient and clinical characteristics by treatment group. Overall, the patient demographics were similar between both groups. The patient population was mostly white, overweight, and the median age in both groups was around 63 . Tobacco use was significantly lower $(P=.02)$ in the fibrin glue group however there was no significant difference in Type II diabetes, COPD or steroid use. There was no statistical difference in cancer type or stage amongst patients however, the majority had colorectal cancer and Stage IV disease. The operative time was significantly longer in the experimental versus the control group (360 mins vs. 507 mins, $\mathrm{p}=0.005)$ and over $80 \%$ of the cases were open elective procedures involving midline incisional hernias.

Table 2 suggests that the odds of having a hernia recurrence is four times higher in the trans fascial suture group, compared to the fibrin glue fixation group, but this was not statistically significant (p-value $=0.35 ; \mathrm{OR}=4.00 ; 95 \% \mathrm{CI}$ : $0.41-39.37$ ). Assuming a non-information beta $(0.5,0.5)$ prior, we computed the Bayesian posterior probability of hernia recurrence in each group, which suggests that there is a $90 \%$ probability that the hernia recurrence rate is higher in the trans fascial group (control) compared to the fibrin glue fixation group (experimental). (Figure 1)

In terms of seroma formation, there was no statistically significant difference in seroma formation requiring IR drainage between the two groups (Table 3, p-value=1.00; $\mathrm{OR}=1.80 ; 95 \% \mathrm{CI}: 0.15$ - 21.57). Again, assuming a non-information beta $(0.5,0.5)$ prior, we computed the Bayesian posterior probability of seroma formation in each group, which suggests that there is a $67 \%$ probability that the seroma formation rate is higher in the trans fascial group (control) compared to the fibrin glue fixation group (experimental). (Figure 2)

Figure 3 shows the Kaplan-Meier estimates of overall survival (OS) by treatment group. The median OS was 29.1 months in the control group and was not reached yet for the experimental group. There was no statistically significant difference in OS between the two groups $(\mathrm{p}=0.34)$.

Table 1. Summary of patient and clinical characteristics by treatment group.

\begin{tabular}{|c|c|c|c|}
\hline Characteristics & $\begin{array}{l}\text { Transfascial suture } \\
(\text { control) group }(n=22)\end{array}$ & $\begin{array}{l}\text { Fibrin glue (experimental) } \\
\text { group }(n=19)\end{array}$ & $P$ value \\
\hline \multicolumn{4}{|l|}{ Demographics } \\
\hline Age (y), median (range) & $63(35-85)$ & $64(39-84)$ & .99 \\
\hline BMI $\left(\mathrm{kg} / \mathrm{m}^{2}\right)$, median (range) & $31.1(23.7-56.3)$ & $30.9(21.7-41.2)$ & .59 \\
\hline Operative time (minutes), median (range) & $360.5(107-787)$ & $507.5(118-1081)$ & .005 \\
\hline Hospital length of stay (days), median (range) & $4(0-14)$ & $5(3-10)$ & .18 \\
\hline \multicolumn{4}{|l|}{ Sex, No. (\%) } \\
\hline Female & $8(36.4)$ & $10(52.6)$ & .36 \\
\hline Male & $14(63.6)$ & $9(47.4)$ & \\
\hline \multicolumn{4}{|l|}{ Race/ethnicity, No. (\%) } \\
\hline White & $13(59.1)$ & $17(89.5)$ & \\
\hline African American & $2(9.1)$ & $0(0)$ & .05 \\
\hline Asian/Pacific Islander & $2(9.1)$ & $2(10.5)$ & \\
\hline Hispanic & $5(22.7)$ & $0(0)$ & \\
\hline \multicolumn{4}{|l|}{ Comorbidities } \\
\hline \multicolumn{4}{|l|}{ Type II Diabetes mellitus, No. (\%) } \\
\hline No & $16(72.7)$ & $15(78.9)$ & .73 \\
\hline Yes & $6(27.3)$ & $4(21.1)$ & \\
\hline \multicolumn{4}{|l|}{ Tobacco use, No. (\%) } \\
\hline No & $14(63.6)$ & $18(94.7)$ & .02 \\
\hline Yes & $8(36.4)$ & $1(5.3)$ & \\
\hline \multicolumn{4}{|l|}{ Cancer stage, No. (\%) } \\
\hline I & $1(4.5)$ & $1(7.1)$ & \\
\hline II & $4(33.3)$ & $2(14.3)$ & .28 \\
\hline III & $3(25)$ & $1(7.1)$ & \\
\hline IV & $4(33.3)$ & $10(71.4)$ & \\
\hline \multicolumn{4}{|l|}{ Previous abdominal XRT, No. (\%) } \\
\hline No & $17(77.3)$ & $12(66.7)$ & .50 \\
\hline Yes & $5(22.7)$ & $6(33.3)$ & \\
\hline \multicolumn{4}{|l|}{ Previous chemotherapy, No. (\%) } \\
\hline No & $6(27.3)$ & $3(16.7)$ & .48 \\
\hline Yes & $16(72.7)$ & $15(83.3)$ & \\
\hline \multicolumn{4}{|l|}{ COPD No. (\%) } \\
\hline No & $21(95.5)$ & $19(100)$ & 1.00 \\
\hline Yes & $1(4.5)$ & $0(0)$ & \\
\hline \multicolumn{4}{|l|}{ Steroid use, No. (\%) } \\
\hline No & $22(100)$ & $18(94.7)$ & .46 \\
\hline Yes & $0(0)$ & $1(5.3)$ & \\
\hline
\end{tabular}




\begin{tabular}{|c|c|c|c|}
\hline Characteristics & $\begin{array}{l}\text { Transfascial suture } \\
\text { (control) group }(n=22)\end{array}$ & $\begin{array}{l}\text { Fibrin glue (experimental) } \\
\text { group }(n=19)\end{array}$ & $P$ value \\
\hline \multicolumn{4}{|c|}{ Previous incisional hernia repair, No. (\%) } \\
\hline No & $18(81.8)$ & $15(78.9)$ & \multirow[t]{3}{*}{1.00} \\
\hline Yes & $4(18.2)$ & $4(21.1)$ & \\
\hline \multicolumn{3}{|l|}{ Perioperative Factors } & \\
\hline \multicolumn{4}{|l|}{ Incision location, No. (\%) } \\
\hline Midline & $18(81.8)$ & $16(84.2)$ & \multirow{4}{*}{.20} \\
\hline Stoma & $1(4.5)$ & $1(5.3)$ & \\
\hline Umbilical & $3(13.6)$ & $0(0)$ & \\
\hline Stoma/Midline & $0(0)$ & $2(10.5)$ & \\
\hline \multicolumn{4}{|l|}{ Drains placed, No. (\%) } \\
\hline No & $7(31.8)$ & $1(5.3)$ & \multirow[t]{2}{*}{.05} \\
\hline Yes & $15(68.2)$ & $18(94.7)$ & \\
\hline \multicolumn{4}{|l|}{ Surgical procedure, No. (\%) } \\
\hline Lap & $1(4.5)$ & $0(0)$ & \multirow{3}{*}{.09} \\
\hline Robotic & $0(0)$ & $3(15.8)$ & \\
\hline Open & $21(95.5)$ & $16(84.2)$ & \\
\hline \multicolumn{4}{|c|}{ Use of advancement flaps, No. (\%) } \\
\hline No & $17(77.3)$ & $14(73.7)$ & \multirow[t]{2}{*}{1.00} \\
\hline Yes & $5(22.7)$ & $5(26.3)$ & \\
\hline \multicolumn{4}{|l|}{ Type of advancement flap, No. (\%) } \\
\hline Anterior/component/separation & $2(9.1)$ & $0(0)$ & \multirow[t]{2}{*}{.44} \\
\hline TAR & $3(13.6)$ & $5(26.3)$ & \\
\hline \multicolumn{4}{|l|}{ Surgery type, No. (\%) } \\
\hline Emergent & $2(9.1)$ & $0(0)$ & \multirow[t]{2}{*}{.49} \\
\hline Elective & $20(90.9)$ & $19(100)$ & \\
\hline \multicolumn{4}{|c|}{ Surgical case involving additional 2 or more surgical services, No. (\%) } \\
\hline No & $16(72.7)$ & $12(63.2)$ & \multirow[t]{2}{*}{.74} \\
\hline Yes & $6(27.3)$ & $7(36.8)$ & \\
\hline \multicolumn{4}{|c|}{ Post-operative complications } \\
\hline \multicolumn{4}{|c|}{ Need for IR drainage of seroma, No. (\%) } \\
\hline No & $20(90.9)$ & $18(94.7)$ & \multirow[t]{2}{*}{1.00} \\
\hline Yes & $2(9.1)$ & $1(5.3)$ & \\
\hline \multicolumn{4}{|l|}{ Hernia recurrence, No. (\%) } \\
\hline No & $18(81.8)$ & $18(94.7)$ & \multirow[t]{2}{*}{.35} \\
\hline Yes & $4(18.2)$ & $1(5.3)$ & \\
\hline \multicolumn{4}{|l|}{ SSI, No. (\%) } \\
\hline No & $19(86.4)$ & $16(84.2)$ & \multirow[t]{2}{*}{1.00} \\
\hline Yes & $3(13.6)$ & $3(15.8)$ & \\
\hline \multicolumn{4}{|l|}{ 30-day readmission, No. (\%) } \\
\hline No & $20(90.9)$ & $16(84.2)$ & \multirow[t]{2}{*}{.65} \\
\hline Yes & $2(9.1)$ & $3(15.8)$ & \\
\hline
\end{tabular}

A $P$ value of 0.5 was considered statistically significant.

Abbreviations: BMI, body mass index; COPD, chronic obstructive pulmonary disease; IR, interventional radiology; SSI, superficial surgical site infection; TAR, transversus abdominus release; XRT, radiotherapy.

Table 2. Association between experimental/control group and hernia recurrence.

\begin{tabular}{lll}
\hline & Hernia Recurrence=No & Hernia Recurrence=Yes \\
\hline Control & 18 & $4(18 \%)$ \\
Experimental & 18 & $1(5 \%)$ \\
\hline
\end{tabular}

p-value $=0.35$ (Fisher's exact test)

Odds Ratio $=4.00(95 \%$ CI: $0.41-39.37)$ for control vs. experimental group

Table 3. Association between experimental/control group and the need for IR drainage for seroma.

\begin{tabular}{lll}
\hline & need for IR drainage for seroma=No & need for IR drainage for seroma=Yes \\
\hline Control & 20 & $2(9 \%)$ \\
Experimental & 18 & $1(5 \%)$ \\
\hline
\end{tabular}

p-value $=1.00$ (Fisher's exact test)

Odds Ratio=1.80 (95\% CI: $0.15-21.57)$ for control vs. experimental group 

Incisional Hernia Repair with Transfascial Sutures vs. Fibrin Glue for Mesh Fixation

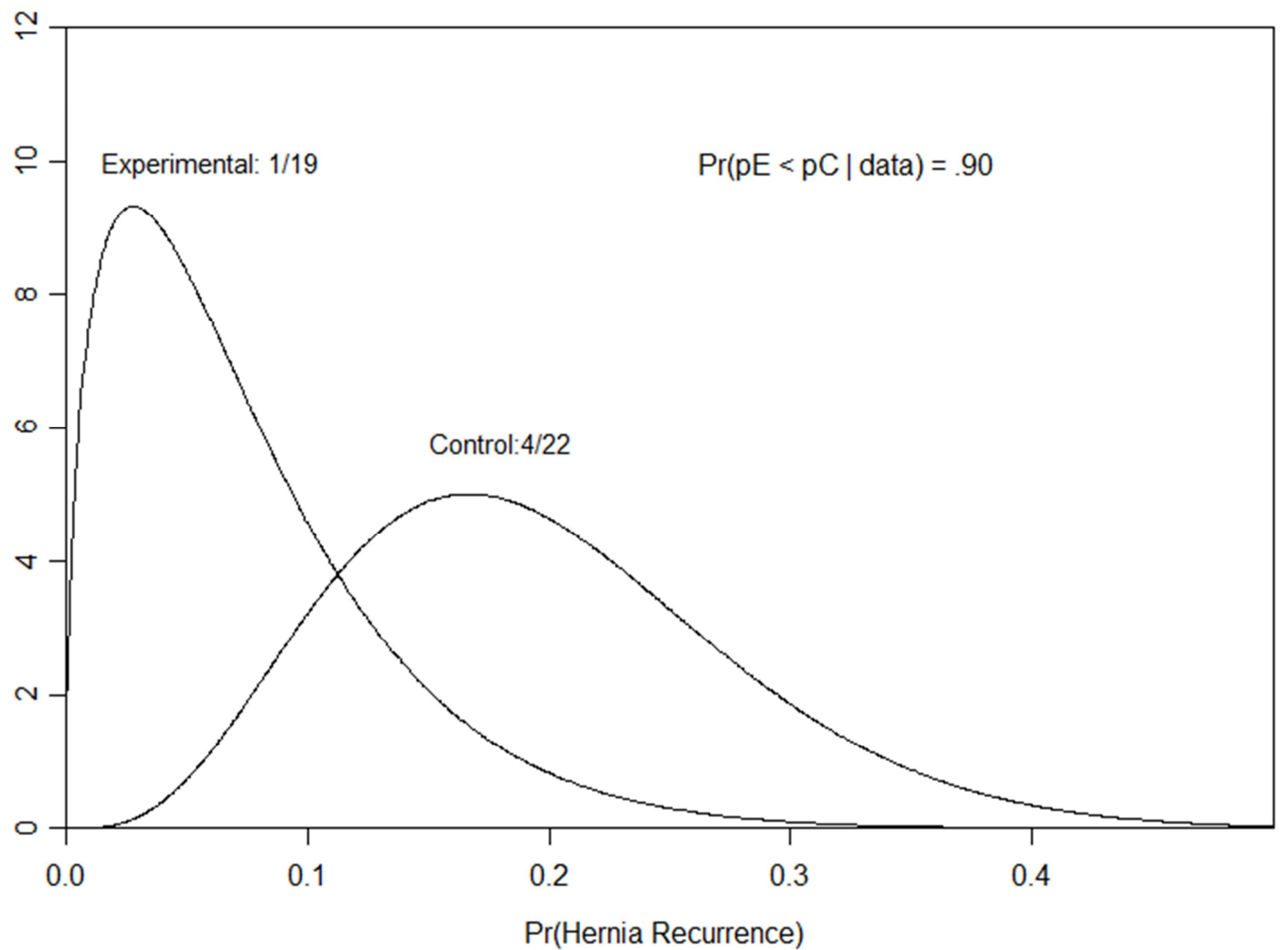

Figure 1. Posterior distribution of the probability of hernia recurrence in the experiment vs. control group. pE is the probability of hernia recurrence in the experimental group; $p C$ is the probability of hernia recurrence in the control group.

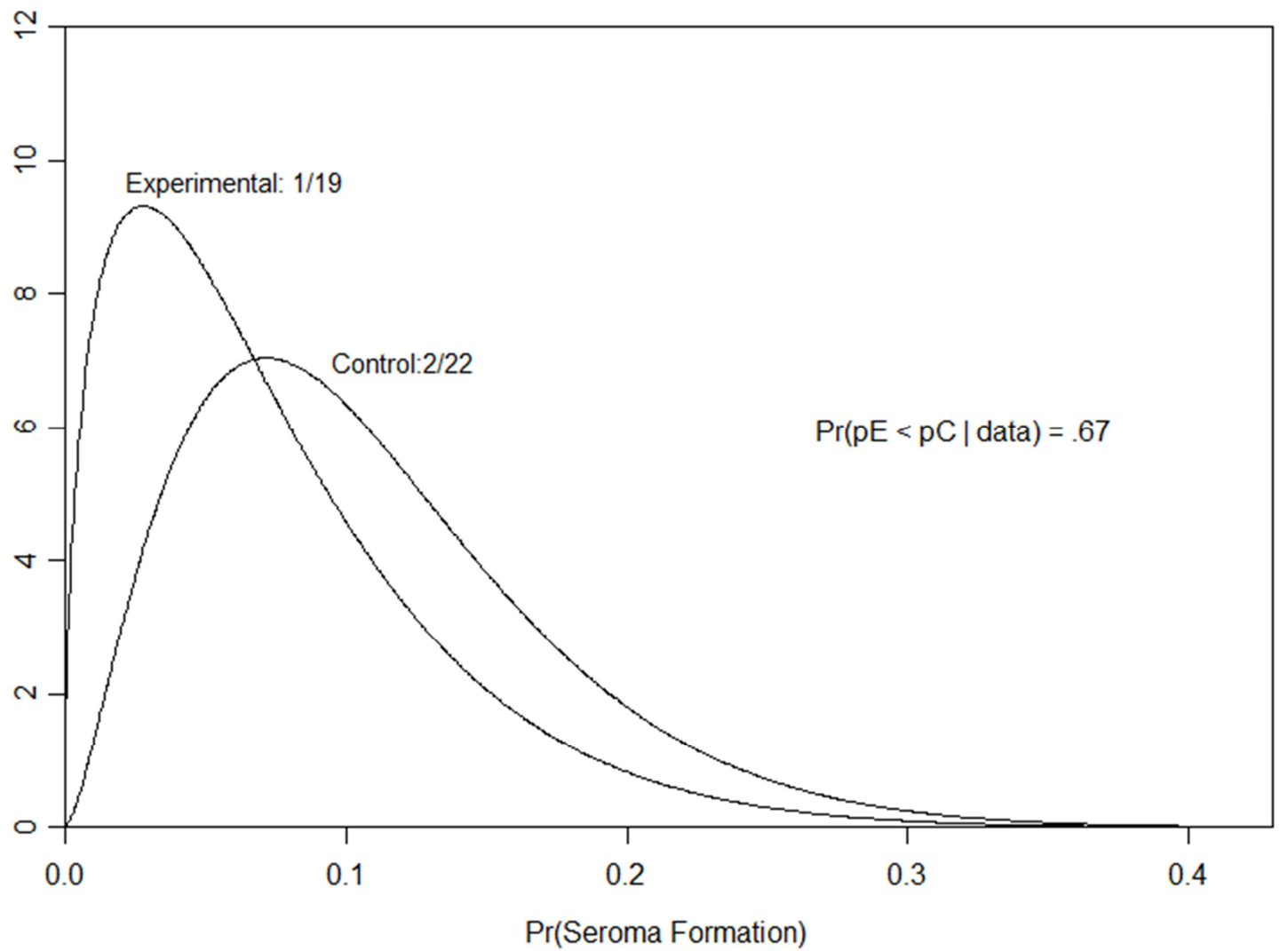

Figure 2. Posterior distribution of the probability of seroma formation in the experiment vs. control group. pE is the probability of seroma formation in the experimental group; $p C$ is the probability of seroma formation in the control group. 


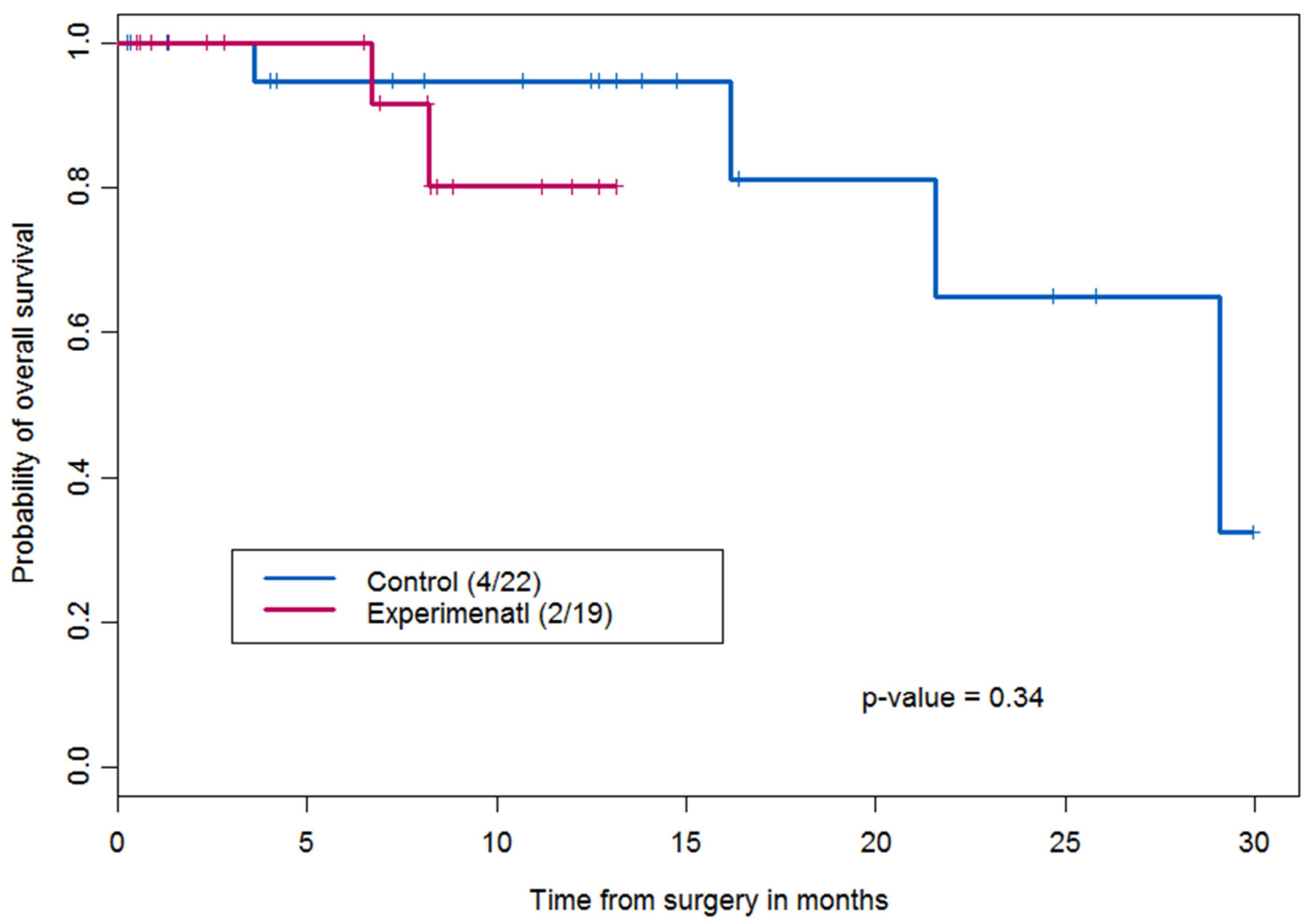

Figure 3. Kaplan-Meier estimates of the probabilities of overall survival by treatment group.

\section{Discussion}

Our study demonstrated that patients who undergo Rives-Stoppa ventral incisional hernia repair do not have an increased risk of hernia recurrence or seroma formation when undergoing surgery with fibrin glue versus transfascial suture for mesh fixation. Specifically, Bayesian analysis demonstrated the probability of hernia recurrence was $90 \%$ and seroma formation requiring intervention was $67 \%$ in the transfascial group in comparison to the fibrin glue group. In addition, there was no evidence of wound dehiscence or death with the use of fibrin glue for retrorectus hernia repair.

In animal models, both sutures and fibrin glue demonstrated similar mesh-fixation strengths after one week [11]. This could account for the similar rates of hernia recurrence we observed in the fibrin glue and transfascial suture mesh fixation groups. Moazzez and Dubina found that, in retrorectus repairs with Tisseel fibrin sealant, tension was taken off the posterior sheath by facilitating the posterior rectus fascia's contact with most of the mesh rather than with only a few spots. The latter situation is typical when transfascial sutures are used or when no fixation method is applied. Thus, fibrin glue use results in reduced tension in the posterior midline and reduced risk of fascial breakdown. [12]

The general impact of fibrin glue on seroma formation can be explained by its sealing of small blood vessels and lymphatic vessels, which results in reduced fluid retention. In addition, sealing tissue-to-wound surfaces decreases retromuscular dead space, thus enabling faster tissue revascularization. Prior studies have examined the effect of fibrin glue use on postoperative seroma rates following abdominal wall surgery. However, these data were limited to small case series. In a study evaluating patients who underwent laparoscopic ventral hernia repair, the use of fibrin glue was associated with a $28 \%$ reduction in seroma formation by post-operative day 7 and a $46 \%$ reduction 1 month after surgery [13]. In another study of 60 patients who underwent open ventral hernia repair, the use of fibrin glue helped prevent seroma formation and decreased the rate of wound complications [14]. Similarly, Férnandez et al. noted substantially less local morbidity and shorter hospital stays among patients who had had fibrin glue applied following incisional hernia repair with dermolipectomy. [15] Our data suggests that there was no difference in readmission or length of stay in the fibrin glue group when compared to the transfascial suture group.

Our study has some inherent limitations. The short-term follow-up was an accepted limitation of the study, as our goal was to report early outcomes and potential practicalities of using the Rives-Stoppa technique with fibrin glue for mesh fixation. It is also noteworthy that all surgeries in this study were performed by the same 2 surgeons, so it is possible that the improvements in postoperative outcomes were partially attributable to improvements in operative technique. The most obvious limitation may be a limited sample size which made our study underpowered to detect classical statistical differences. Although we may not have had enough patients to detect statistical difference using traditional statistical methods because of power. We addressed this by doing a Bayesian analysis. This is innovative and turns the weakness of the paper into a strength. Because it answers the clinical question of whether recurrence or seroma forms with a probability that can be updated when we introduce new 
practice changes. In addition, the high probability of lower recurrence and seroma formation requiring intervention argues that there is a potential advantage to fibrin sealant fixation, and our hypothesis generating retrospective study could serve as evidence for a formal RCT to determine causation.

\section{Conclusion}

Fibrin glue provides an alternative to transfascial sutures for the fixation of retromuscular mesh during ventral incisional hernia repairs performed using the Rives-Stoppa technique with no difference in hernia recurrence rate or seroma requiring intervention. The probability that fibrin sealant is superior to transfascial sutures for recurrence and seroma requiring operative intervention is high. Our retrospective study is hypothesis generating because it has biologic plausibility, high probability of clinical benefit, and deserves an adequately powered prospective study such as a randomized trial. Future studies in the form of prospective, randomized clinical trials are needed to assess the long-term outcomes of mesh fixation with fibrin glue.

\section{Funding}

Supported by the NIH/NCI under award number P30CA016672 and used the Biostatistics Resource Group.

\section{Conflicts of Interest}

The authors declare that they have no competing interests.

\section{Acknowledgements}

MD Anderson Research Medical Library Editing Services

\section{References}

[1] Santora, T. A. and J. J. Roslyn, Incisional hernia. Surg Clin North Am, 1993.73 (3): p. 557-70.

[2] Pauli, E. M. and M. J. Rosen, Open ventral hernia repair with component separation. Surg Clin North Am, 2013. 93 (5): p. 1111-33.
[3] Millikan, K. W., Incisional hernia repair. Surg Clin North Am, 2003. 83 (5): p. 1223-34.

[4] El-Gazzaz, G., et al., Risk of infection and hernia recurrence for patients undergoing ventral hernia repair with non-absorbable or biological mesh during open bowel procedures. Tech Coloproctol, 2013. 17 (3): p. 315-20.

[5] Gronnier, C., et al., Risk factors for chronic pain after open ventral hernia repair by underlay mesh placement. World $\mathrm{J}$ Surg, 2012. 36 (7): p. 1548-54.

[6] McLanahan, D., et al., Retrorectus prosthetic mesh repair of midline abdominal hernia. Am J Surg, 1997. 173 (5): p. 445-9.

[7] Vermeulen, J., I. Alwayn, and L. P. Stassen, Prolonged abdominal wall pain caused by transfascial sutures used in the laparoscopic repair of incisional hernia. Surg Endosc, 2003. 17 (9): p. 1497.

[8] Chevrel, J. a. R. A., The Use of Fibrin Glues in the Surgical Treatment of Incisional Hernias. Hernia 1997. 1: p. 9-14.

[9] Rhemtulla, I., et al. Retromuscular Mesh Repair Using Fibrin Glue: Early Outcomes and Cost-effectiveness of an Evolving Technique. Plastic Reconstr Surg Glob Open, 2019. Apr; 7 (4): e2184

[10] Chandra, P., D. Phalgune, and S. Shah, Comparison of the Clinical Outcome and Complications in Laparoscopic Hernia Repair of Inguinal Hernia With Mesh Fixation Using Fibrin Glue vs Tacker. Indian J Surg, 2016. 78 (6): p. 464-470.

[11] Fortelny, R. H., et al., Use of fibrin sealant (Tisseel/Tissucol) in hernia repair: a systematic review. Surg Endosc, 2012. 26 (7): p. $1803-12$.

[12] Moazzez, A. and E. D. Dubina, A Novel Approach to Mesh Fixation in Retrorectus Ventral Hernia Repair Using Fibrin Sealant. J Am Coll Surg, 2017. 225 (3): p. e1-e4.

[13] Morales-Conde, S., et al., Influence of fibrin sealant in preventing postoperative seroma and normalizing the abdominal wall after laparoscopic repair of ventral hernia. Surg Endosc, 2013.27 (9): p. 3214-9.

[14] Kohler, G., et al., Prevention of subcutaneous seroma formation in open ventral hernia repair using a new low-thrombin fibrin sealant. World J Surg, 2014. 38 (11): p. 2797-803.

[15] Fernández Lobato, R., et al., Tissucol application in dermolipectomy and incisional hernia repair. Int Surg, 2001. 86 (4): p. $240-5$. 